\title{
Signal Detection of Potentially Drug-Induced Acute Liver Injury in Children Using a Multi-Country Healthcare Database Network
}

\author{
Carmen Ferrajolo $\cdot$ Preciosa M. Coloma - Katia M. C. Verhamme • Martijn J. Schuemie • \\ Sandra de Bie - Rosa Gini • Ron Herings • Giampiero Mazzaglia • Gino Picelli • Carlo Giaquinto • \\ Lorenza Scotti - Paul Avillach • Lars Pedersen - Francesco Rossi - Annalisa Capuano • \\ Johan van der Lei · Gianluca Trifiró • Miriam C. J. M. Sturkenboom · EU-ADR consortium
}

Published online: 21 January 2014

(C) Springer International Publishing Switzerland 2014

\begin{abstract}
Background Data mining in spontaneous reporting databases has shown that drug-induced liver injury is infrequently reported in children.

Objectives Our objectives were to (i) identify drugs potentially associated with acute liver injury (ALI) in children and adolescents using electronic healthcare record (EHR) data; and (ii) to evaluate the significance and novelty of these associations.
\end{abstract}

Electronic supplementary material The online version of this article (doi:10.1007/s40264-013-0132-9) contains supplementary material, which is available to authorized users.

C. Ferrajolo $(\varangle)$. P. M. Coloma · K. M. C. Verhamme .

M. J. Schuemie - S. de Bie · J. van der Lei · G. Trifiró .

M. C. J. M. Sturkenboom

Department of Medical Informatics, Erasmus University

Medical Center, Dr. Molewaterplein 50, 3015 GE Rotterdam,

The Netherlands

e-mail: c.ferrajolo@erasmusmc.nl; carmen.ferrajolo@unina2.it

C. Ferrajolo - F. Rossi - A. Capuano

Pharmacology Section, Department of Experimental Medicine,

Campania Regional Center of Pharmacovigilance and

Pharmacoepidemiology, Second University of Naples, Via L. De

Crecchio, 7, 80138 Naples, Italy

R. Gini

Regional Health Agency of Tuscany, Florence, Italy

R. Herings

PHARMO Institute, Utrecht, The Netherlands

G. Mazzaglia

Italian College of General Practitioners, Florence, Italy

G. Picelli

Pedianet-Società Servizi Telematici SRL, Padua, Italy
Methods We identified potential cases of ALI during exposure to any prescribed/dispensed drug for individuals $<18$ years old from the EU-ADR network, which includes seven databases from three countries, covering the years 1996-2010. Several new methods for signal detection were applied to identify all statistically significant associations between drugs and ALI. A drug was considered statistically significantly associated with ALI, using all other time as a reference category, if the $95 \%$ CI lower band of the relative risk was $>1$ and in the presence of at least three exposed cases of ALI. Potentially new signals were distinguished from already known associations concerning ALI (whether in adults and/or in the paediatric population)

C. Giaquinto

Department of Paediatrics, University Hospital of Padua, Padua, Italy

L. Scotti

Department of Statistics and Quantitative Methods, University of Milano-Bicocca, Milan, Italy

P. Avillach

LESIM, ISPED, University of Bordeaux 2, Bordeaux, France

L. Pedersen

Department of Clinical Epidemiology, Aarhus University

Hospital, Aarhus, Denmark

G. Trifiró

Department of Clinical and Experimental Medicine, University of Messina, Messina, Italy

M. C. J. M. Sturkenboom

Epidemiology, Erasmus University Medical Center, Rotterdam, The Netherlands 
through manual review of published literature and drug product labels.

Results The study population comprised 4,838,146 individuals aged $<18$ years, who contributed an overall 25,575,132 person-years of follow-up. Within this population, we identified 1,015 potential cases of ALI. Overall, 20 positive drug-ALI associations were detected. The associations between ALI and domperidone, flunisolide and human insulin were considered as potentially new signals. Citalopram and cetirizine have been previously described as hepatotoxic in adults but not in children, while all remaining associations were already known in both adults and children.

Conclusions Data mining of multiple EHR databases for signal detection confirmed known associations between ALI and several drugs, and identified some potentially new signals in children that require further investigation through formal epidemiologic studies. This study shows that EHRs may complement traditional spontaneous reporting systems for signal detection and strengthening.

\section{Introduction}

Drug-induced acute liver injury (ALI) is more likely to occur in the post-marketing than the pre-marketing setting, as the incidence is low. This is particularly true in children, since they are not frequently included in clinical trials and, if included, follow-up is usually short. In an earlier publication, based on data from the World Health Organisation (WHO) spontaneous reporting system (SRS) database, we showed that liver toxicity in children is infrequently reported as an adverse drug reaction (only $1 \%$ of total reports in the paediatric population), with paracetamol (acetaminophen), anticonvulsants and anti-tuberculosis agents being the most frequently implicated drugs [1]. However, it is well known that the reporting of ADRs is strongly underestimated $[1,2]$. In the aftermath of the rofecoxib safety concerns, several initiatives in both the USA and the EU started to explore the use of routinely collected data from electronic healthcare records (EHRs) as a source for signal detection/refinement [3-7]. The EU-ADR Project (Exploring and Understanding Adverse Drug Reactions by Integrative Mining of Clinical Records and Biomedical Knowledge [http://www.euadr-project.org]) is a collaboration of 18 public and private institutions in the EU representing academic research, general practice, healthcare services administration and pharmaceutical industry that has produced an integrated system of EHR databases for drug safety surveillance [8]. The large population coverage of the EU-ADR network makes it particularly suitable for drug safety signal detection in special subpopulations such as the paediatric population, but its potential in this setting has not yet been studied.

The aim of this study was to identify drugs associated with ALI in children and adolescents in the EU-ADR database network. We further investigated these potential signals by scrutinizing whether these drug-ALI associations have been previously reported in the adult and/or paediatric population.

\section{Materials and Methods}

\subsection{Setting}

Healthcare data from 1 January 1996 to 31 December 2010 were retrieved from the EU-ADR database network, which has been described in depth in previous publications $[8,9]$. For this study, we used only paediatric data from seven European EHR and claims databases originating from three countries. Health-Search/CSD LPD (HSD) and Pedianet from Italy and Integrated Primary Care Information (IPCI) from the Netherlands are population-based electronic medical record databases that include demographic and clinical information. The Aarhus University Hospital Database (Aarhus, Denmark), PHARMO research database (the Netherlands) and the regional Italian databases of Lombardy and of Tuscany (Agenzia Regionale di Sanità della Toscana, ARS) are all comprehensive record-linkage systems in which drug dispensing data of a well defined population are linked to hospital discharge diagnoses and other registries collecting clinical information (e.g. laboratory tests). Most healthcare services, including pharmacy services and hospitalizations are covered by the national health system in Italy and Denmark and by obligatory health insurance in the Netherlands. In all of these countries, general practitioners (GPs) or family paediatricians serve as 'gatekeepers' of the healthcare system.

\subsection{Cohort Definition and Follow-Up Time}

The study population included all children and adolescents younger than 18 years who were registered within one of the above databases for at least 1 year. This 1-year requirement was waived for newborns and infants younger than 1 year during the study period whose follow-up started immediately at date of birth or at their respective registration. Follow-up of all other patients started from cohort entry until the first occurrence of any of the following events, whatever came first: first diagnosis of ALI; transferring out of the practice; 18th birthday; death; or end of the study period (31 December 2010). 


\subsection{Acute Liver Injury (ALI) Ascertainment}

Both diagnostic codes and laboratory values, whenever available, were used to ascertain potential cases of ALI in different databases. As these databases use different terminologies for coding medical diagnoses and contain different types of information, a process of translation of coding algorithms in different databases was set up, which was based on identification of ALI-related medical concepts in the Unified Medical Language System (UMLS) and projection of these codes into different terminologies: (i) International Classification of Primary Care (ICPC) for IPCI; (ii) International Classification of Diseases 9th revision-Clinical Modification (ICD-9 CM) for ARS, HSD, Lombardy, Pedianet and PHARMO; and (iii) ICD-10th revision for Aarhus. IPCI, HSD, and Pedianet also explored free text within the clinical narratives, using specific keywords relevant to ALI, as well as pertinent laboratory examinations, whenever available [10, 11]. The UMLS codes and coding algorithm for ALI are listed in the Electronic Supplementary Material.

To prevent finding spurious associations, potential cases of ALI due to other specified causes, such as viral infections, hepatic neoplasm, autoimmune hepatitis, genetic and metabolic disorder-related hepatopathy (e.g. hemochromatosis, $\alpha 1$-antitrypsin deficiency, Wilson Disease, Gilbert Syndrome) and biliary tract diseases, were not included.

\subsection{Drug Exposure}

Drug exposure was assessed using data from prescriptions or pharmacy dispensing. Because of the nature of these databases, exposure to over-the-counter medications, such as paracetamol, is not captured. All databases code drugs using the WHO Anatomical Therapeutic Chemical (ATC) classification system. The number of person-years (PY) of exposure was calculated per single compound (ATC 5th level). The duration covered by each prescription/dispensing was estimated by the legend duration (if dosing regimen was available) or based on the defined daily dose (DDD) [12].

\subsection{Data Analysis}

Data on patient demographics, clinical events (i.e. ALI), and prescriptions were locally generated from each database and formatted towards a simple common data model [13]. Based on the common data model, data were aggregated by databases using a custom-built software, Jerboa $\odot$ [9].

In the EU-ADR, several statistical methods have been developed and tested for signal detection. Based on a reference set (i.e. surrogate gold standard) and performance analysis, the best performing method was the Longitudinal
Gamma Poisson Shrinker (LGPS) [14], which is an adaptation of a method used for signal detection in SRS, i.e. Gamma Poisson Shrinker (GPS). LGPS estimates the age- and sexadjusted incidence rate ratios (RR) during the exposure of interest against all other follow-up time (on other drugs and off drugs) as reference while additionally applying Bayesian shrinkage $\left(\mathrm{RR}_{\mathrm{LGPS}}\right)[14,15]$. All the drugs for which we observed at least three exposed cases of ALI and with a lower $95 \% \mathrm{CI}$ of $\mathrm{RR}_{\mathrm{LGPS}}>1$ were considered as potential signals [16]. In a sensitivity analysis, we compared the risk estimates derived from LGPS with the estimates from the self-controlled case series (SCCS) method. Confounding by indication (or contraindication) may arise when a drug treatment serves as a marker for a clinical characteristic or medical condition that triggers the use of the treatment and that, at the same time, increases the risk of the outcome under study. In SCCS analysis, cases serve as their own controls, thereby allowing time-fixed confounding factors, known and unknown (e.g. confounding by indication), to be controlled for implicitly [14]. As in the main analysis, a drug-ALI association was considered as statistically significant if the lower $95 \%$ CI of the RR, calculated using SCCS, was $>1$ with at least three exposed cases.

In addition, for each potential drug-ALI signal, we evaluated the possible role of protopathic bias by applying LEOPARD (Longitudinal Evaluation of Observational Profiles of Adverse events Related to Drugs) [14]. Since protopathic bias occurs when a drug is prescribed for an early manifestation of a disease that has not yet been diagnosed, the number of the specific drug prescriptions initiated increases after the event date (relative to the period prior to the event date), indicating that the drug is used to treat prodromal symptoms of the event, rather than cause it. Accordingly, for every suspect drug-ALI association, LEOPARD compared the prescription rates within a fixed window of 25 days prior to and 25 days after the occurrence of ALI. Thus, the method flagged the drug-ALI associations as potentially due to protopathic bias if the prescription rate after the event was higher than the prescription rate before the event [15].

For all signals that could not be explained by protopathic bias, based on current scientific evidence, we assessed whether the association was known, unknown or incompletely documented and we also evaluated the possible biological plausibility. For this purpose, we reviewed primary drug-related information sources: (i) Micromedex (http://www.thomsonhc.com/hcs/librarian) and (ii) the summary of product characteristics (SPCs), derived from the following two databases: (i) electronic Medicines Compendium (eMC, http://www.medicines.org.uk/emc) and (ii) FarmaDati (http://www.farmadati.it). With regard to the SPCs, we specifically looked at the sections 'Undesirable/adverse effects' and 'Special warnings and 


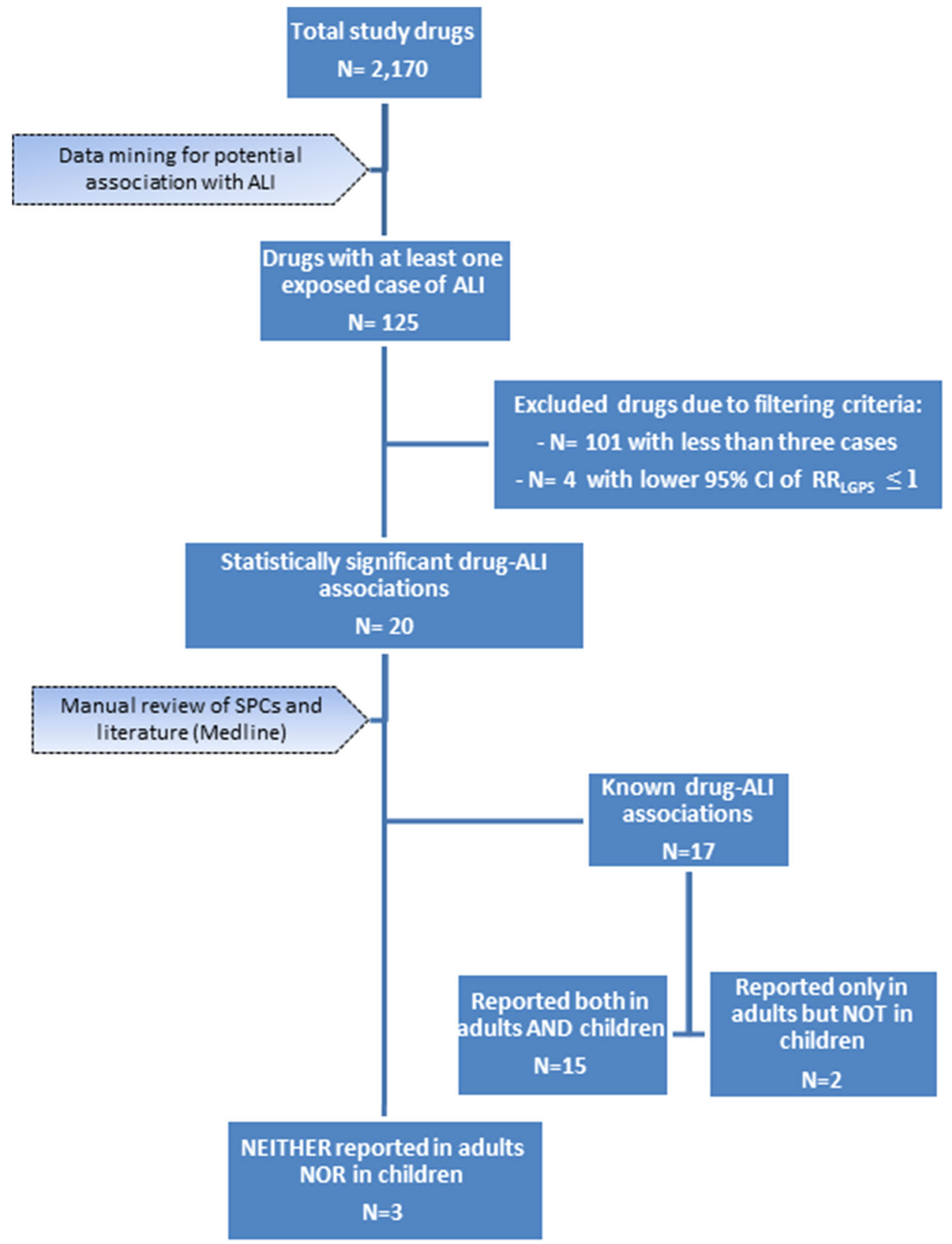

Fig. 1 Schematic diagram of the identification of potentially new signals of ALI in children and adolescents. Note: statistically significant association means drugs with at least three exposed cases of ALI and a lower band of $95 \%$ CI of RR >1 when applying the LGPS method. ALI acute liver injury, LGPS Longitudinal Gamma Poisson Shrinker, SPC summary of product characteristics

precautions'. We additionally explored whether the drugALI associations were already described in the published literature (via MEDLINE: web.ebscohost.com) in adults and/or more specifically in children.

\section{Results}

The paediatric population of the EU-ADR network overall comprised 4,838,146 children and adolescents (aged
$<18$ years) contributing 25,575,132 PYs of follow-up in the period 1996-2010. Among 1,015 potential cases of ALI identified in this database network, 251 potential cases (24.7\%) occurred during exposure to any drug $(1,032,899$ PY) accounting for a crude incidence of $2.4 / 10,000$ PY (2.1-2.7) among exposed.

The stepwise procedure leading to identification of potential signals is schematically shown in Fig. 1. Of 2,170 drugs prescribed/dispensed to the paediatric population during the study period, almost $6 \%(N=125)$ were 
involved in at least one potential case of ALI. However, only for 20 drugs were at least three exposed cases observed across all databases (Table 1). Potential ALI cases occurred most often during use of anti-infectives for systemic use $(N=6)$ and drugs for alimentary tract and metabolism $(N=5)($ Table 1$)$.

\subsection{Detection of Statistically Significant Drug-ALI Associations}

The drugs that were associated with ALI, using unexposed period to the drug of interest as reference, are shown in Table 2. Ranked by the strength of the $\mathrm{RR}_{\mathrm{LGPS}}$ for ALI, the top ten drugs included metoclopramide, methotrexate, azathioprine, prednisolone, ranitidine, diclofenac, omeprazole, domperidone, phenoxymethylpenicillin, i.e. penicillin V, and human insulin. Applying the SCCS method, most of these drugs remained significantly associated with ALI, except for insulin, cefaclor and flunisolide. The magnitude of risk decreased substantially for the anti-ulcer drugs (ranitidine and omeprazole), the corticosteroids (prednisolone and prednisone) and the immunosuppressants (azathioprine and methotrexate), pointing towards confounding by indication. The association became stronger for metoclopramide, domperidone, diclofenac, valproic acid and erythromycin (Table 2). Notably, the associations for insulin, cefaclor and flunisolide were not confirmed using this method only because of lack of power, although the potential risk remained high. Based on the results derived from LEOPARD, ten of the drugs with statistically significant associations using LGPS and SCCS were classified as potentially due to protopathic bias (Table 2).

\subsection{Evaluation of the Significance and Novelty of the Signals}

Table 3 describes the available knowledge on the 20 statistically significant drug-ALI associations we observed. Three associations were identified as a potentially new signal (i.e. they had not been previously described in the literature, either in adults or in children): domperidone, human insulin and flunisolide. For these drugs, there is currently no mention of ALI as a possible adverse event, either in the SPCs or in the published literature, irrespective of the age group. In addition, two other drugs, citalopram and cetirizine, have not thus far been described as associated with hepatotoxicity in the paediatric population, although ALI has been documented in adults and is reported in the SPCs.

\section{Discussion}

In this study, three previously undocumented signals of ALI in children and adolescents were identified using 'realworld' data from a combination of multiple European healthcare databases.

Among all the drugs being prescribed/dispensed in children and adolescents during the study period, around $1 \%(20 / 2,170)$ was potentially associated with ALI. ALI occurred most frequently during use of antibacterial agents and drugs for peptic ulcer and gastroesophageal reflux disease. These data are very much in line with previous findings from an analysis of the WHO SRS [1].

According to the definition of safety signals by Hauben and Aronson [17], not all statistically significant
Table 1 Drugs used in the paediatric population and those associated with acute liver injury

\footnotetext{
${ }^{a}$ Drugs with at least three exposed cases and a value with lower band of $95 \%$ CI of $\mathrm{RR}_{\text {LGPS }}>1$
}

\begin{tabular}{|c|c|c|}
\hline $\begin{array}{l}\text { Main anatomic group } \\
\text { (WHO ATC Classification, I level) }\end{array}$ & $\begin{array}{l}\text { Study drugs } \\
N=2,170\end{array}$ & $\begin{array}{l}\text { No. of drugs with statistically } \\
\text { significant associations }{ }^{\mathrm{a}} \\
\text { (\% within anatomic group) }\end{array}$ \\
\hline (A) Alimentary \& metabolism & 391 & $5(1.3)$ \\
\hline (N) Nervous system & 269 & $2(0.7)$ \\
\hline (J) Anti-infectives for systemic use & 232 & $6(2.6)$ \\
\hline (D) Dermatologicals & 203 & - \\
\hline (C) Cardiovascular system & 192 & - \\
\hline (S) Sensory organs & 169 & - \\
\hline (R) Respiratory system & 160 & $2(1.3)$ \\
\hline (G) Genito-urinary and sex hormones & 153 & - \\
\hline (M) Musculo-skeletal system & 104 & $1(1.0)$ \\
\hline (B) Blood and blood forming organs & 97 & - \\
\hline (L) Antineoplastics and immunomodulators & 76 & $2(2.6)$ \\
\hline (H) Systemic hormonal preparations & 72 & $2(2.8)$ \\
\hline (P) Antiparasitic & 35 & - \\
\hline (V) Various & 17 & - \\
\hline
\end{tabular}


Table 2 Comparison of different methods applied for signal detection concerning acute liver injury

\begin{tabular}{|c|c|c|c|c|c|c|c|}
\hline ATC & Drugs (ATC 5th level) & $\begin{array}{l}\text { No. of } \\
\text { cases }\end{array}$ & $\begin{array}{l}\text { Exposure } \\
\text { (PYs) }\end{array}$ & $\begin{array}{l}\text { Crude IR/ } \\
10,000 \\
\text { PY }(95 \% \text { CI })\end{array}$ & $\begin{array}{l}\mathrm{RR}_{\mathrm{LGPS}}(95 \% \\
\mathrm{CI})\end{array}$ & $\begin{array}{l}\mathrm{RR}_{\mathrm{SCCS}}(95 \% \\
\mathrm{CI})\end{array}$ & LEOPARD $^{b}$ \\
\hline \multirow[t]{2}{*}{ A02 } & Ranitidine (A02BA02) & 7 & $3,833.86$ & $\begin{array}{l}18.3 \\
(8.14-35.8)\end{array}$ & $\begin{array}{l}43.7 \\
(17.7-87.6)\end{array}$ & $\begin{array}{l}12.9 \\
(4.9-34.0)\end{array}$ & Yes \\
\hline & Omeprazole (A02BC01) & 7 & $5,583.97$ & $\begin{array}{l}12.5 \\
(5.6-24.6)\end{array}$ & $\begin{array}{l}29 \\
(9.5-60.9)\end{array}$ & $\begin{array}{l}13.3 \\
(4.9-35.6)\end{array}$ & Yes \\
\hline \multirow[t]{2}{*}{ A03 } & Metoclopramide(A03FA01) & 4 & 282.27 & $\begin{array}{l}141.7 \\
(47.4-336.9)\end{array}$ & $\begin{array}{l}262.8 \\
(85.7-636.2)\end{array}$ & $\begin{array}{l}449.1 \\
(104.0-1,938.7)\end{array}$ & No \\
\hline & Domperidone (A03FA03) & 3 & $2,903.41$ & $\begin{array}{l}10.3 \\
(2.1-30.2)\end{array}$ & $\begin{array}{l}22.9 \\
(3.5-89.2)\end{array}$ & $\begin{array}{l}35.9 \\
(10.5-122.1)\end{array}$ & No \\
\hline A10 & Insulin, human ${ }^{\mathrm{a}}(\mathrm{A} 10 \mathrm{AC} 01)$ & 3 & $3,344.73$ & $\begin{array}{l}9.0 \\
(1.8-26.2)\end{array}$ & $\begin{array}{l}9.2 \\
(2.3-41.2)\end{array}$ & $\begin{array}{l}4.1 \\
(0.8-20.4)\end{array}$ & No \\
\hline \multirow[t]{2}{*}{ H02 } & Prednisolone (H02AB06) & 5 & $1,699.56$ & $\begin{array}{l}29.4 \\
(11.2-64.5)\end{array}$ & $\begin{array}{l}45.1 \\
(12.3-104.2)\end{array}$ & $\begin{array}{l}6.0 \\
(1.9-19.6)\end{array}$ & Yes \\
\hline & Prednisone (H02AB07) & 3 & $5,647.87$ & $\begin{array}{l}5.3 \\
(1.5-14.2)\end{array}$ & $\begin{array}{l}8.9 \\
(2.3-39.4)\end{array}$ & $\begin{array}{l}5.6 \\
(1.8-17.9)\end{array}$ & Yes \\
\hline \multirow[t]{3}{*}{ J01C } & Amoxicillin (J01CA04) & 10 & $59,842.54$ & $\begin{array}{l}1.7 \\
(8.6-29.6)\end{array}$ & $\begin{array}{l}3.9 \\
(2.1-6.8)\end{array}$ & $\begin{array}{l}4.2 \\
(2.3-7.5)\end{array}$ & No \\
\hline & $\begin{array}{l}\text { Phenoxymethylpenicillin } \\
\text { (J01CE02) }\end{array}$ & 14 & $6,623.26$ & $\begin{array}{l}21.1 \\
(12.1-34.5)\end{array}$ & $\begin{array}{l}17 \\
(8.6-30.0)\end{array}$ & $\begin{array}{l}16.7 \\
(9.9-28.1)\end{array}$ & No \\
\hline & $\begin{array}{l}\text { Amoxicillin/clavulanate } \\
\text { (J01CR02) }\end{array}$ & 9 & $81,268.6$ & $\begin{array}{l}1.1 \\
(0.5-2.0)\end{array}$ & $\begin{array}{l}3.5 \\
(1.8-6.3)\end{array}$ & $\begin{array}{l}2.7 \\
(1.4-5.0)\end{array}$ & Yes \\
\hline J01D & Cefaclor $^{\mathrm{a}}$ (J01DC04) & 3 & $15,857.28$ & $\begin{array}{l}1.9 \\
(0.5-5.1)\end{array}$ & $\begin{array}{l}3.6 \\
(1.2-8.8)\end{array}$ & $\begin{array}{l}2.6 \\
(0.8-9.3)\end{array}$ & No \\
\hline \multirow[t]{2}{*}{$\mathrm{J} 01 \mathrm{~F}$} & Erythromycin (J01FA01) & 4 & $3,722.42$ & $\begin{array}{l}10.7 \\
(3.6-25.5)\end{array}$ & $\begin{array}{l}6.9 \\
(2.4-21.1)\end{array}$ & $\begin{array}{l}12.3 \\
(4.1-37.2)\end{array}$ & No \\
\hline & Clarithromycin (J01FA09) & 5 & $36,597.8$ & $\begin{array}{l}1.4 \\
(0.5-3.0)\end{array}$ & $\begin{array}{l}4 \\
(1.7-8.3)\end{array}$ & $\begin{array}{l}6.8 \\
(3.3-13.9)\end{array}$ & No \\
\hline \multirow[t]{2}{*}{ L04 } & Methotrexate (L04AX03) & 8 & 840.8 & $\begin{array}{l}35.7 \\
(9.9-95.2)\end{array}$ & $\begin{array}{l}211.3 \\
(98.8-401.5)\end{array}$ & $\begin{array}{l}180.1 \\
(20.7-1568.3)\end{array}$ & Yes \\
\hline & Azathioprine (L04AX01) & 3 & 618.99 & $\begin{array}{l}129.2 \\
(61.0-243.9)\end{array}$ & $\begin{array}{l}48.6 \\
(5.8-153.3)\end{array}$ & $\begin{array}{l}4.8 \\
(1.1-21.1)\end{array}$ & Yes \\
\hline M01 & Diclofenac (M01AB05) & 5 & $2,290.91$ & $\begin{array}{l}21.8 \\
(8.3-47.8)\end{array}$ & $\begin{array}{l}31.2 \\
(7.3-76.8)\end{array}$ & $\begin{array}{l}39.8 \\
(16.7-94.6)\end{array}$ & Yes \\
\hline N03 & Valproic acid (N03AG01) & 4 & $12,502.01$ & $\begin{array}{l}3.2 \\
(1.1-7.6)\end{array}$ & $\begin{array}{l}5.1 \\
(1.9-12.3)\end{array}$ & $\begin{array}{l}24.4 \\
(5.4-111.0)\end{array}$ & No \\
\hline N06 & Citalopram (N06AB04) & 3 & $2,878.31$ & $\begin{array}{l}10.4 \\
(2.9-27.8)\end{array}$ & $\begin{array}{l}5.6 \\
(1.8-17.6)\end{array}$ & $\begin{array}{l}7.6 \\
(1.2-50.5)\end{array}$ & Yes \\
\hline R03 & Flunisolide $^{\mathrm{a}}$ (R03BA03) & 4 & $27,548.87$ & $\begin{array}{l}1.5 \\
(0.5-34.5)\end{array}$ & $\begin{array}{l}3.4 \\
(1.3-7.6)\end{array}$ & $\begin{array}{l}2.7 \\
(0.9-8.1)\end{array}$ & No \\
\hline R06 & Cetirizine (R06AE07) & 5 & $43,255.13$ & $\begin{array}{l}1.2 \\
(0.4-2.5)\end{array}$ & $\begin{array}{l}2.5 \\
(1.0-5.1)\end{array}$ & $\begin{array}{l}3.0 \\
(1.2-7.7)\end{array}$ & Yes \\
\hline
\end{tabular}

$A L I$ acute liver injury, LEOPARD Longitudinal Evaluation of Observational Profiles of Adverse events Related to Drugs, $L G P S$ Longitudinal Gamma Poisson Shrinker, $P Y$ person-year, SCCS self-controlled case series

Drugs with at least three exposed cases of ALI and a lower band of $95 \%$ CI of RR $>1$ when applying LGPS method

${ }^{a}$ Not statistically significant association when using SCCS method

${ }^{\mathrm{b}}$ Yes = protopathic bias is likely to be present, no = protopathic bias is unlikely to be present 
Table 3 Novelty of statistically significant drug-acute liver injury associations

\begin{tabular}{|c|c|c|c|}
\hline \multirow[t]{2}{*}{ Drugs } & \multirow[t]{2}{*}{$\mathrm{SPCs}^{\mathrm{a}}$} & \multicolumn{2}{|c|}{ Literature (MEDLINE) ${ }^{\mathrm{b}}$} \\
\hline & & Adults & Children $^{c}$ \\
\hline Ranitidine & Yes & Yes & Yes \\
\hline Omeprazole & Yes & Yes & Yes \\
\hline Metoclopramide $^{\mathrm{a}}$ & No & Yes & Yes \\
\hline Domperidone & No & No & No \\
\hline Insulin (human) & No & No & No \\
\hline Prednisolone & Yes & Yes & Yes \\
\hline Prednisone & Yes & Yes & Yes \\
\hline Amoxicillin & Yes & Yes & Yes \\
\hline Phenoxymethylpenicillin & Yes & Yes & Yes (in co-therapy with erythromycin) \\
\hline Amoxicillin/clavulanate & Yes & Yes & Yes \\
\hline Cefaclor & Yes & No & Yes \\
\hline Erythromycin & Yes & Yes & Yes \\
\hline Clarithromycin & Yes & Yes & Yes \\
\hline Azathioprine & Yes & Yes & Yes \\
\hline Methotrexate & Yes & Yes & Yes \\
\hline Diclofenac & Yes & Yes & Yes \\
\hline Valproic acid & Yes & Yes & Yes \\
\hline Citalopram $^{\mathrm{e}}$ & Yes & Yes & No \\
\hline Flunisolide & No & No & No \\
\hline Cetirizine & Yes & Yes & No \\
\hline
\end{tabular}

$A L I$ acute liver injury, SPC summary of product characteristics

a SPCs reviewed: (i) Micromedex (http://www.thomsonhc.com/hcs/librarian); (ii) electronic Medicines Compendium (eMC, http://www. medicines.org.uk/emc); and (iii) FarmaDati (http://www.farmadati.it)

b Literature (via MEDLINE: web.ebscohost.com)

c Yes $=$ drug-ALI association was reported also or only in children

${ }^{\mathrm{d}}$ Use not approved for children $<16$ years

${ }^{\mathrm{e}}$ Use not approved for children and adolescents $<18$ years

associations should be regarded as potential new signals. To detect whether each drug-ALI association (i.e. possible signal) was newly discovered, we performed a manual review of the SPCs and other primary drug information sources including Micromedex and MEDLINE and scientific literature. To the best of our knowledge, three of the drug-ALI associations were not previously described in the literature, either in adults or in children, and were also not labeled in the SPCs. These drugs were domperidone, flunisolide and insulin (human). Two other drug-ALI associations (citalopram, cetirizine) were not previously described in children but have already been described in adults.

\subsection{Potentially New Signals}

The association between domperidone and ALI was identified as a potential new signal. However, prodromal signs/ symptoms of liver injury such as nausea and vomiting represent the main indication for domperidone intake. Therefore, although LEOPARD did not automatically flag this as protopathic bias, we cannot exclude that this is a spurious association due to protopathic bias. If indeed the interval between incriminated prodromal symptoms and onset of event is larger than 25 days, protopathic bias will not be detected by LEOPARD [14].

Flunisolide is a synthetic inhaled corticosteroid with potent topical anti-inflammatory activity, with an oral bioavailability ranging from 7 to $20 \%[18,19]$. After gastrointestinal and lung absorption, the drug undergoes rapid and extensive first-pass metabolism by the liver to an inactive 6-beta-hydroxylated metabolite. Systemic effects have not been reported for the commonly used doses. Higher doses of flunisolide, as well as the other inhaled corticosteroids, may result in adverse events similar to those occurring during the corticosteroid systemic use due to an increased oral adsorption of the medicine. Although signs/symptoms of liver injury are reported as undesirable side effects with the 
use of all systemic corticosteroids, liver injury is not specifically mentioned for inhaled corticosteroids, including flunisolide. Differences in pharmacokinetic characteristics across inhaled corticosteroids might suggest differences in the occurrence of adverse drug reactions [20]. Further investigations comparing inhaled corticosteroids, specifically concerning hepatotoxicity, are required.

Insulin therapy is indicated in children and adolescents with type 1 diabetes and in children with ketosis or diabetic ketoacidosis when the distinction between type of diabetes is unclear. There are no reports in the literature associating human insulin with (acute) liver injury. However, it is possible that insulin induces undesired weight gain from hunger triggered by insulin-induced hypoglycemia. Comparative trials of patients with type 2 diabetes found that weight gain and risk of hypoglycemia might occur during the use of human insulin more than with the analogues [21, 22]. Moreover, weight gain could lead to hepatic steatosis, an aetiopathologic sign of non-alcoholic fatty liver disease (NAFLD), explaining the potential role of human insulin in this type of liver injury [23]. On the other hand, since NAFLD has been reported to increase the risk of type 2 diabetes [24], and potentially the use of insulin, the role of insulin is unclear in this association.

The associations identified for flunisolide and human insulin deserve a separate discussion because these associations were not confirmed by SCCS analysis, suggesting the influence of potential confounder factors on the estimations. This seems particularly true for insulin, for which the nature (causal inference) of the connection to NAFLD remains a matter of speculation [25]. However, although not significant (meaning that we had insufficient power for this statistical test only because of the low prevalence of exposure), the associations were high for flunisolide $\left(\mathrm{RR}_{\mathrm{SCCS}}=2.7\right)$ and human insulin $\left(\mathrm{RR}_{\mathrm{SCCS}}=4.1\right)$. Accordingly, we still cannot rule out that these drugs are truly associated with an increased risk of ALI and the associations of ALI, but these drugs should be investigated in a formal pharmacoepidemiological study in a wider paediatric setting in order to confirm or confute these potential signals.

\subsection{Potentially New Signals in Paediatrics}

Citalopram is a widely used selective serotonin reuptake inhibitor (SSRI). Rare instances of acute, clinically apparent episodes of liver injury with moderate or marked liver enzyme elevations with or without jaundice have been described in less than $1 \%$ of adults within 6-10 weeks, or earlier, of citalopram therapy. One prospective study investigated the use of central nervous system (CNS) agents and risk of idiosyncratic drug-induced liver injury in children but did not identify citalopram as a suspect drug [26].
Cetirizine is a second-generation antihistamine used for the treatment of allergic rhinitis, angioedema and chronic urticaria. Cetirizine and analogues have been related to rare, isolated cases of clinically apparent ALI, with a pattern ranging from cholestatic hepatitis to hepatocellular jaundice in adults [27-30]. Nevertheless, as urticaria may represent a prodromic sign of underlying liver disease, potential protopathic bias as an alternative explanation for the association cannot be excluded and is supported by the results from LEOPARD.

Although the association between ALI and metoclopramide has already been described in two epidemiologic studies, including within the paediatric population, showing a risk of liver injury for metoclopramide ranging from moderate to low [31, 32], we also believe that here, the potential of protopathic bias holds similar to that described for domperidone.

Finally, data mining on EHR databases detected associations for several drugs already widely known as hepatotoxic in both adults and children [33]. The application of the LGPS as main analysis and the SCCS as sensitivity analysis suggested that the system can produce reliable results.

In fact, when applying the SCCS method, the associations from LGPS were amplified or reduced, but still remained for all drugs already known to be hepatotoxic, except for cefaclor. Similarly, using LEOPARD to filter signals due to protopathic bias improved the overall performance of signal detection. LEOPARD flagged associations with anti-acid drugs, such as ranitidine or omeprazole, as spurious. Indeed, these drugs might have been prescribed for gastric discomfort, which is one of the prodromal signs of hepatic injury. On the other hand, it was rather unexpected that LEOPARD was not able to detect this bias for metoclopramide or domperidone. This might be explained by the \pm 25 -day window that LEOPARD (by default) applies as described above. Protopathic bias was correctly captured for azathioprine, methotrexate, diclofenac and systemic corticosteroids. Indeed, these drugs are prescribed for conditions (such as rheumatic disease) associated with ALI.

\subsection{Strengths and Limitations}

The main strength of this study is its capability to (retrospectively) observe a large number of children and adolescents in a 'real-world' setting by combining data from multiple longitudinal healthcare databases. While we were able to investigate the associations between ALI and the most frequently used drugs in children in Europe [34], the system did not allow the exploration of over-the-counter medications (such as paracetamol), in-hospital used drugs or less frequently prescribed drugs (such as anti- 
tuberculosis agents and other anti-convulsants) that are well known to be hepatotoxic in children. For instance, we found a non-statistically significant increase in risk of ALI related to the use of paracetamol, or anti-convulsants (i.e. carbamazepine, phenobarbital, vigabatrin or gabapentin) or other CNS agents (i.e. atomoxetine, risperidone, sertraline, tramadol or methadone). This result does not imply the absence of association, but rather the low prevalence of exposure of these drugs in such prescribing/dispensing registries.

We used harmonised database-specific disease codes and free text search to automatically identify liver injury from the database network. Individual causality assessment of the identified associations was not conducted. However, a previous study using a USA database network demonstrated that outcome misclassification does not influence the results concerning signal detection [35]. Usually, exclusion of alternative causes for the potential signal is part of an aetiology-based approach for the evaluation of a physician-reported ADR. To reproduce this process using EHR databases, we used the SCCS method to control for time-fixed confounders such as genetic factors, socio-economic status, individual frailty and severity of underlying disease [14].

To investigate the potential of protopathic bias, we used the LEOPARD method. Despite using filtering criteria for significance and sensitivity analyses, the likelihood of obtaining false-positive results cannot be excluded and further validation of the newly identified associations needs to be carried out [36].

\section{Conclusions}

We found potentially new signals concerning ALI for flunisolide, domperidone and insulin. There was also a signal for ALI in children for citalopram and cetirizine, but this association has already been described in adults. All potentially new signals require further evaluation in hypothesis-testing studies (e.g. formal pharmacoepidemiologic studies) to better account for bias and confounding. Our findings highlight the potential of electronic healthcare databases to complement traditional SRS for drug safety signal detection and strengthening in a paediatric setting. However, combining data from other longitudinal healthcare and paediatric-specific databases would be meaningful to gain sufficient statistical power to investigate a large range of drugs specifically used in children and adolescents.

Acknowledgments This research has been funded by the European Commission Seventh Framework Programme (FP7/2007-2013) under grant no. 215847-the EU-ADR Project. The funding agency had no role in the design and conduct of the study, the collection and management of data, the analysis or interpretation of the data or preparation, review, or approval of the manuscript.

Conflicts of interest Martijn J. Schuemie has become an employee of Janssen R\&D since completing this research and has received a grant from the Foundation for the National Institutes of Health (FNIH). Katia M.C. Verhamme received unconditional research grants from Pfizer, Boehringer-Ingelheim, Novartis and Yamanouchi. None of these are related to the content of this research. Carmen Ferrajolo, Preciosa M. Coloma, Sandra de Bie, Rosa Gini, Giampiero Mazzaglia, Gino Picelli, Carlo Giaquinto, Lorenza Scotti, Paul Avillach, Lars Pedersen, Francesco Rossi, Annalisa Capuano, Johan van der Lei, Ron Herings, Miriam C.J.M. Sturkenboom and Gianluca Trifiro declare that they have no conflicts of interest directly relevant to the content of this study.

\section{References}

1. Ferrajolo C, Capuano A, Verhamme KM, Schuemie M, Rossi F, Stricker BH, et al. Drug-induced hepatic injury in children: a case/non-case study of suspected adverse drug reactions in VigiBase. Br J Clin Pharmacol. 2010;70(5):721-8.

2. Stephenson WP, Hauben M. Data mining for signals in spontaneous reporting databases: proceed with caution. Pharmacoepidemiol Drug Saf. 2007;16(4):359-65.

3. Coloma PM, Avillach P, Salvo F, Schuemie MJ, Ferrajolo C, Pariente A, et al. A reference standard for evaluation of methods for drug safety signal detection using electronic healthcare record databases. Drug Saf. 2013;36(1):13-23.

4. Schneeweiss S, Avorn J. A review of uses of health care utilization databases for epidemiologic research on therapeutics. J Clin Epidemiol. 2005;58(4):323-37.

5. Platt R, Wilson M, Chan KA, Benner JS, Marchibroda J, McClellan M. The new Sentinel Network-improving the evidence of medical-product safety. N Engl J Med. 2009;361(7): $645-7$.

6. Stang PE, Ryan PB, Racoosin JA, Overhage JM, Hartzema AG, Reich $\mathrm{C}$, et al. Advancing the science for active surveillance: rationale and design for the Observational Medical Outcomes Partnership. Ann Intern Med. 2010;153(9):600-6.

7. Trifiro G, Pariente A, Coloma PM, Kors JA, Polimeni G, Miremont-Salame G, et al. Data mining on electronic health record databases for signal detection in pharmacovigilance: which events to monitor? Pharmacoepidemiol Drug Saf. 2009;18(12):1176-84.

8. Trifiro G, Fourrier-Reglat A, Sturkenboom MC, Diaz Acedo C, Van Der Lei J. The EU-ADR project: preliminary results and perspective. Stud Health Technol Inform. 2009;148:43-9.

9. Coloma PM, Schuemie MJ, Trifiro G, Gini R, Herings R, Hippisley-Cox J, et al. Combining electronic healthcare databases in Europe to allow for large-scale drug safety monitoring: the EUADR Project. Pharmacoepidemiol Drug Saf. 2011;20(1):1-11.

10. Avillach P, Joubert M, Thiessard F, Trifiro G, Dufour JC, Pariente A, et al. Design and evaluation of a semantic approach for the homogeneous identification of events in eight patient databases: a contribution to the European EU-ADR project. Stud Health Technol Inform. 2010;160(Pt 2):1085-9.

11. Avillach P, Mougin F, Joubert M, Thiessard F, Pariente A, Dufour JC, et al. A semantic approach for the homogeneous identification of events in eight patient databases: a contribution to the European eu-ADR project. Stud Health Technol Inform. 2009;150:190-4. 
12. Updated 4 April 2012; http://www.whocc.no/atc_ddd_index/.

13. Avillach P, Coloma PM, Gini R, Schuemie M, Mougin F, Dufour $\mathrm{JC}$, et al. Harmonization process for the identification of medical events in eight European healthcare databases: the experience from the EU-ADR project. J Am Med Inform Assoc. 2013;20(1):184-92.

14. Schuemie MJ. Methods for drug safety signal detection in longitudinal observational databases: LGPS and LEOPARD. Pharmacoepidemiol Drug Saf. 2011;20(3):292-9.

15. Schuemie MJ, Coloma PM, Straatman H, Herings RM, Trifiro G, Matthews JN, et al. Using electronic health care records for drug safety signal detection: a comparative evaluation of statistical methods. Med Care. 2012;50(10):890-7.

16. Slattery J, Alvarez Y, Hidalgo A. Choosing thresholds for statistical signal detection with the proportional reporting ratio. Drug Saf. 2013;36(8):687-92.

17. Hauben M, Aronson JK. Defining 'signal' and its subtypes in pharmacovigilance based on a systematic review of previous definitions. Drug Saf. 2009;32(2):99-110.

18. Kantar A, Mroueh S, Fiocchi A. A reappraisal of the clinical efficacy of nebulized flunisolide in pediatric asthma: the Italian experience. Allergy Asthma Proc. 2007;28(6):671-87.

19. Kelly HW. Establishing a therapeutic index for the inhaled corticosteroids: part I. Pharmacokinetic/pharmacodynamic comparison of the inhaled corticosteroids. J Allergy Clin Immunol. 1998;102(4 Pt 2):S36-51.

20. Todd GR, Acerini CL, Ross-Russell R, Zahra S, Warner JT, McCance D. Survey of adrenal crisis associated with inhaled corticosteroids in the United Kingdom. Arch Dis Child. 2002;87(6):457-61.

21. Handelsman Y, Mechanick JI, Blonde L, Grunberger G, Bloomgarden ZT, Bray GA, et al. American Association of Clinical Endocrinologists Medical Guidelines for Clinical Practice for developing a diabetes mellitus comprehensive care plan. Endocr Pract. 2011;17(Suppl 2):1-53.

22. Inzucchi SE, Bergenstal RM, Buse JB, Diamant M, Ferrannini E, Nauck M, et al. Management of hyperglycemia in type 2 diabetes: a patient-centered approach: position statement of the American Diabetes Association (ADA) and the European Association for the Study of Diabetes (EASD). Diabetes Care. 2012;35(6):1364-79.

23. Powell EE, Cooksley WG, Hanson R, Searle J, Halliday JW, Powell LW. The natural history of nonalcoholic steatohepatitis: a follow-up study of forty-two patients for up to 21 years. Hepatology. 1990;11(1):74-80.
24. Bugianesi E, Moscatiello S, Ciaravella MF, Marchesini G. Insulin resistance in nonalcoholic fatty liver disease. Curr Pharm Des. 2010;16(17):1941-51.

25. Marchesini G, Brizi M, Morselli-Labate AM, Bianchi G, Bugianesi E, McCullough AJ, et al. Association of nonalcoholic fatty liver disease with insulin resistance. Am $\mathrm{J}$ Med. 1999;107(5):450-5.

26. Molleston JP, Fontana RJ, Lopez MJ, Kleiner DE, Gu J, Chalasani N. Characteristics of idiosyncratic drug-induced liver injury in children: results from the DILIN prospective study. J Pediatr Gastroenterol Nutr. 2011;53(2):182-9.

27. Ekiz F, Yuksel I, Ekiz O, Coban S, Basar O, Yuksel O. Levocetirizine induced hepatotoxicity in a patient with chronic urticaria. Ann Hepatol. 2011;10(2):237-8.

28. Jurawan R, Smith A. Severe hepatitis in a primary sclerosing cholangitis patient receiving recent cetirizine therapy. N Z Med J. 2010;123(1309):106-7.

29. Rodriguez-Gomez SJ, Zamora-Martinez T, Bailador-Andres C, Fuentes-Coronel AM, Martin-Arribas MI. Severe intrahepatic cholestasis associated with cetirizine. Gastroenterol Hepatol. 2009;32(5):383-4.

30. Pompili M, Basso M, Grieco A, Vecchio FM, Gasbarrini G, Rapaccini GL. Recurrent acute hepatitis associated with use of cetirizine. Ann Pharmacother. 2004;38(11):1844-7.

31. Sabate M, Ibanez L, Perez E, Vidal X, Buti M, Xiol X, et al. Risk of acute liver injury associated with the use of drugs: a multicentre population survey. Aliment Pharmacol Ther. 2007;25(12): 1401-9.

32. de Abajo FJ, Montero D, Madurga M, Garcia Rodriguez GA. Acute and clinically relevant drug-induced liver injury: a population based case-control study. Br J Clin Pharmacol. 2004; 58(1):71-80.

33. Ferrajolo C, Verhamme KM, Trifiro G, 't Jong GW, Giaquinto C, Picelli G, et al. Idiopathic acute liver injury in paediatric outpatients: incidence and signal detection in two European countries. Drug Saf. 2013;36(10):1007-16.

34. Sturkenboom MC, Verhamme KM, Nicolosi A, Murray ML, Neubert A, Caudri D, et al. Drug use in children: cohort study in three European countries. BMJ. 2008;337:a2245.

35. Reich CG, Ryan PB, Schuemie MJ. Alternative outcome definitions and their effect on the performance of methods for observational outcome studies. Drug Saf. 2013;36(Suppl 1):S181-93.

36. Walker AM. Orthogonal predictions: follow-up questions for suggestive data. Pharmacoepidemiol Drug Saf. 2010;19(5): 529-32. 